\title{
The Effect of Baseline Rescue Medication Use on Efficacy and Safety of Nebulized Glycopyrrolate Treatment in Patients with COPD from the GOLDEN 3 and 4 Studies
}

This article was published in the following Dove Press journal:

International Journal of Chronic Obstructive Pulmonary Disease

James F Donohue (D)

Ayca Ozol-Godfrey ${ }^{2}$

Thomas Goodin ${ }^{2}$

Shahin Sanjar $\mathbb{I D}^{2}$

'Department of Pulmonary Diseases and Critical Care Medicine, University of North Carolina School of Medicine, Chapel Hill, NC, USA; ${ }^{2}$ Sunovion Pharmaceuticals Inc., Marlborough, MA, USA
Correspondence: James F Donohue Department of Pulmonary Diseases and Critical Care Medicine, University of North Carolina School of Medicine, Chapel Hill, NC 27599, USA

Email James_donohue@med.unc.edu
Purpose: Rescue medication use is common in chronic obstructive pulmonary disease (COPD) patients and tends to increase with symptoms and disease severity. An analysis of baseline rescue medication use was conducted to inform on patient phenotypes and subsequent effects on lung function, symptoms, and safety following 12 weeks of nebulized glycopyrrolate (GLY) $25 \mu \mathrm{g}$ twice daily or placebo in patients with moderate-tovery-severe COPD.

Patients and Methods: Pooled data from the 12-week, placebo-controlled GOLDEN 3 and 4 studies $(n=781)$ were used to assign patients into quarters based on baseline rescue medication use (ie, average puffs-per-day) during the run-in period. Placebo-adjusted trough forced expiratory volume in 1 second $\left(\mathrm{FEV}_{1}\right)$, St. George's Respiratory Questionnaire (SGRQ) total score and EXAcerbations of COPD Tool-Respiratory Symptoms (EXACTRS) total score data were reported; safety was evaluated by reviewing the incidence of adverse events (AEs) and serious AEs (SAEs).

Results: Baseline rescue medication use was a proxy for disease severity, evidenced by decreased lung function, increased health status scores, symptom scores and use of background long-acting $\beta 2$-agonists and inhaled corticosteroids across quarters and treatment groups. Treatment with GLY led to greater improvements from baseline in trough $\mathrm{FEV}_{1}$, SGRQ and EXACT-RS scores compared with placebo in all rescue medication use quarters. Additionally, the SGRQ and EXACT-RS exhibited greater improvement with increased baseline rescue medication use with GLY treatment. In the Q4 patients, SGRQ ( $\geq 4$-unit reduction) or EXACT-RS ( $\geq 2$-unit reduction) responders were significantly greater with GLY compared with placebo. AE and SAE incidences were similar across quartiles.

Conclusion: These results suggest that baseline rescue medication use assessments may be useful in the management of COPD. Treatment with nebulized GLY improved lung function and symptom scores, regardless of baseline rescue medication use. These results support the use of nebulized GLY for the treatment of COPD, independent of baseline rescue medication use.

Keywords: COPD, glycopyrrolate, LAMA, rescue medication use

\section{Introduction}

Chronic obstructive pulmonary disease (COPD) is a progressive disease characterized by persistent respiratory symptoms and airflow limitation. ${ }^{1}$ The use of rescue medications is common among patients with COPD to alleviate respiratory symptoms. ${ }^{1}$ In 
addition, COPD patients often experience exacerbations of their symptoms which are defined as an acute worsening of respiratory symptoms that require additional therapy, ${ }^{1}$ most commonly in the form of rescue, short-acting bronchodilators. The frequency of rescue medication use tends to increase with symptom and disease severity, and is associated with exacerbation risk in patients with moderate-tovery-severe COPD. ${ }^{2}$ In a recent meta-analysis of 46,531 patients from 46 studies, rescue medication use correlated with clinically important COPD outcomes, including trough forced expiratory volume in 1 second $\left(\mathrm{FEV}_{1}\right)$, St. George's Respiratory Questionnaire (SGRQ), and annualized moderate or severe exacerbation rates. ${ }^{3}$ While many studies have assessed the impact of treatment on rescue medication use, there are limited analyses of the impact of the level of rescue medication use at baseline on the efficacy and safety of longacting bronchodilators in patients with COPD. In one such analysis, patient stratification based on baseline use of rescue medications did not impact the efficacy and safety of QVA149 compared with placebo or salmeterol/fluticasone combination. $^{4}$

Glycopyrrolate inhalation solution (GLY; Lonhala ${ }^{\circledR}$, Sunovion Pharmaceuticals, Inc., Marlborough, MA, USA) $25 \mu \mathrm{g}$ twice daily (BID) delivered by the eFlow ${ }^{\circledR}$ Closed System (CS) Nebulizer (Magnair ${ }^{\circledR}$, PARI Pharma GmbH, Starnberg, Germany) ${ }^{5}$ was approved by the US Food and Drug Administration (FDA) for the long-term maintenance treatment of airflow obstruction in patients with COPD in December 2017.6 Data from the 12-week, placebocontrolled Glycopyrrolate for Obstructive Lung Disease via Electronic Nebulizer (GOLDEN) 3 and GOLDEN 4 Phase
III studies (NCT02347761 and NCT02347774, respectively) demonstrated statistically significant and clinically important improvements in pulmonary function and patient-reported outcomes (PROs) with GLY compared with placebo in patients with moderate-to-very-severe COPD. ${ }^{7}$ In this analysis of pooled data from the 12-week GOLDEN 3 and GOLDEN 4 studies, we assessed the impact of baseline rescue medication use on patient's physiologic and symptomatic responses to nebulized GLY compared with placebo.

\section{Patients and Methods}

\section{Study Design}

Details of the GOLDEN 3 and GOLDEN 4 studies have been previously described (Figure 1). ${ }^{7}$ Briefly, in the 12-week, multicenter, placebo-controlled, double-blind studies, subjects $(\mathrm{N}=1293)$ were randomized in a $1: 1: 1$ ratio and received placebo or GLY (25 or $50 \mu \mathrm{g}$ BID), via the eFlow CS Nebulizer; 781 patients who received either GLY $25 \mu \mathrm{g}$ BID or placebo and had baseline rescue medication use data available are included in this analysis. Randomization in each of the studies was stratified by background long-acting beta ${ }_{2}$-agonist (LABA) use (yes/no) and by cardiovascular (CV) risk (high/low). Ipratropium bromide, as supplemental medication, and albuterol (salbutamol), as rescue medication, were permitted. Data for the GLY $50 \mu \mathrm{g}$ BID treatment arm are not presented in this posthoc analysis, but were used in the modeling in dividing the rescue medication data into 4 equal quarters using quartiles. Inclusion of the $50 \mu \mathrm{g}$ BID data in the modeling does not confound the interpretation of the findings from the GLY $25 \mu \mathrm{g}$ BID dose.

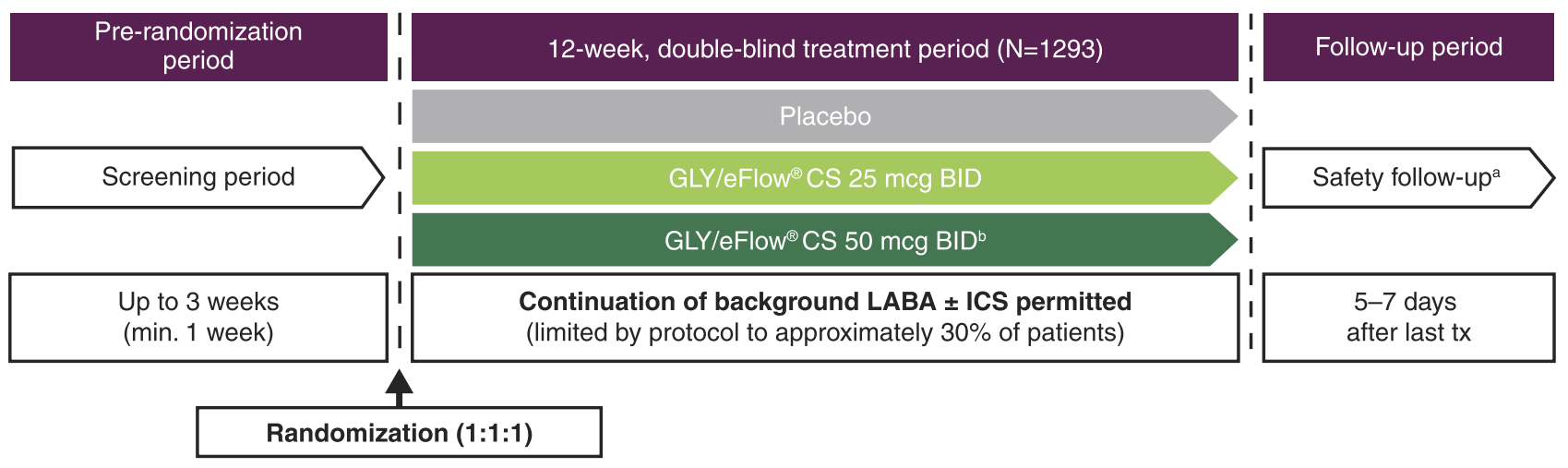

Figure I GOLDEN 3 and GOLDEN 4 study designs: 12-week, randomized, double-blind, placebo-controlled, parallel-group, multicenter studies. ${ }^{7}$

Notes: ${ }^{a}$ SAEs were monitored for 30 days after the last dose of study treatment; ${ }^{b}$ Data for the GLY $50 \mu g$ BID treatment arm are not presented in this post-hoc analysis but were used in the modeling and in defining the rescue medication subgroups studied in this analysis; inclusion of the $50 \mu \mathrm{g} B \mathrm{D}$ data in the modeling does not confound the interpretation of the GLY $25 \mu \mathrm{g}$ BID dose.

Abbreviations: BID, twice daily; CS, closed system; GLY, nebulized glycopyrrolate; GOLDEN, Glycopyrrolate for Obstructive Lung Disease via Electronic Nebulizer; ICS, inhaled corticosteroids; LABA, long-acting beta 2 -agonist; min, minimum; SAE, serious adverse event; tx, treatment. 
The GOLDEN 3 (SUN101-301: project approval number 28481) and GOLDEN 4 (SUN101-302: project approval number 28482) study protocols were approved by Quorum Review IRB North American (US and Canadian) Board (Panel II) prior to patient enrollment, and were conducted in accordance with the protocols, International Council for Harmonization Good Clinical Practice guidelines, and the Declaration of Helsinki. All patients provided written informed consent.

\section{Patients}

Detailed patient inclusion and exclusion criteria have been published previously. ${ }^{7}$ Briefly, enrolled patients included males or females $\geq 40$ years of age, current or ex-smokers with $\geq 10$ pack-year smoking history, a clinical diagnosis of moderate-to-very-severe COPD (as defined by the GOLD 2014 Report $^{8}$ ), and qualifying post-bronchodilator (ipratropium $68 \mu \mathrm{g})$ spirometry $\left(\mathrm{FEV}_{1} \leq 80 \%\right.$ of predicted normal, $\mathrm{FEV}_{1}>0.7 \mathrm{~L}$, and $\mathrm{FEV}_{1}$ /forced vital capacity ratio [FVC] $<0.70)^{7}$

\section{Statistical Analyses}

Pooled patient data from the replicate GOLDEN 3 and GOLDEN 4 studies were grouped into four subgroups using quartiles based on the amount of baseline rescue medication use (Table 1), which was defined as the average number of puffs per day during the 7-day screening period. GLY and placebo treatments were compared in the quartile subgroups for the following endpoints: lung function, as assessed by changes from baseline in trough $\mathrm{FEV}_{1}$ at Week 12; PROs, as measured by changes from baseline in SGRQ total score and EXAcerbations of COPD Tool-Respiratory Symptoms (EXACT-RS) total score at Week 12; rescue medication use, as assessed by changes from baseline in number of daily puffs of rescue medication over 12 weeks. Rescue medication use was recorded daily by patients using an electronic diary. Safety data were analyzed using descriptive statistics. ${ }^{7}$ Adverse events (AEs) and serious adverse events (SAEs) were coded according to MedDRA v15.1 and summarized by treatment, system organ class, and preferred term.

Data are presented for the FDA-approved GLY $25 \mu \mathrm{g}$ BID dose. ${ }^{6}$ Changes from baseline in trough $\mathrm{FEV}_{1}$ and EXACT-RS total score at Week 12 were analyzed using a mixed-model for repeated measures. Changes from baseline in SGRQ total score at Week 12 and changes from baseline in rescue medication use over 12 weeks were analyzed by analysis of covariance. Minimum clinically important differences for the different measures were defined as: reduction in SGRQ total score $\geq 4$ units; ${ }^{9}$ reduction in EXACT-RS total score $\geq 2 .{ }^{10}$ SGRQ responders were analyzed using logistic regression and EXACT-RS responders were analyzed using a longitudinal logistic regression. All models included covariates for baseline level of the appropriate outcome measure, CV risk (high/low), and background LABA use (yes/no). Efficacy analyses used the intent-to-treat (ITT) population and the safety analyses were conducted using the safety population; both populations consisted of all patients randomized to treatment and who received $\geq 1$ dose of study drug. Only data that were measured while on randomized blinded study treatment (ie, on-treatment data) were analyzed. No multiplicity adjustments were made for the comparisons. All $p$-value interpretations are made at the $5 \%$ significance level. All statistical procedures were performed using $\mathrm{SAS}^{\circledR}$ v9.2 or higher (SAS Institute Inc., Cary, NC).

\section{Results}

\section{Patient Demographics and Baseline Characteristics}

Of the 1293 patients in the GOLDEN 3 and GOLDEN 4 studies, 781 patients receiving placebo or GLY $25 \mu \mathrm{g}$ BID were pooled for this analysis; 84 patients (placebo, $n=41$; GLY $25 \mu \mathrm{g}$ BID, $\mathrm{n}=43$ ) did not have baseline rescue medication use data and were removed from the analysis.

Table I Patient Grouping Based on Baseline Rescue Medication Use Quartiles

\begin{tabular}{|c|c|c|c|c|c|c|c|c|}
\hline \multirow[t]{2}{*}{ Rescue Medication Use } & \multicolumn{2}{|l|}{ QI } & \multicolumn{2}{|l|}{ Q2 } & \multicolumn{2}{|l|}{ Q3 } & \multicolumn{2}{|l|}{ Q4 } \\
\hline & $\begin{array}{l}\text { Placebo } \\
n=97\end{array}$ & $\begin{array}{l}\text { GLY } 25 \\
\mu g \text { BID } \\
n=91\end{array}$ & $\begin{array}{l}\text { Placebo } \\
n=|| 3\end{array}$ & $\begin{array}{l}\text { GLY } 25 \mu g \\
\text { BID } \\
n=93\end{array}$ & $\begin{array}{l}\text { Placebo } \\
n=86\end{array}$ & $\begin{array}{l}\text { GLY } 25 \mu g \\
\text { BID } \\
n=\mid / 2\end{array}$ & $\begin{array}{l}\text { Placebo } \\
n=93\end{array}$ & $\begin{array}{l}\text { GLY } 25 \mu g \\
\text { BID } \\
n=96\end{array}$ \\
\hline $\begin{array}{l}\text { Average number of puffs/ } \\
\text { day, median (range) }\end{array}$ & $0.1(0-0.9)$ & $0.1(0-0.9)$ & $1.8(1.0-2.7)$ & $1.8(1.0-2.7)$ & $4.1(2.7-5.2)$ & $3.9(2.8-5.2)$ & $6.6(5.3-14.1)$ & $6.8(5.2-10.4)$ \\
\hline
\end{tabular}

Abbreviations: BID, twice daily; GLY, nebulized glycopyrrolate; Q, quarter. 
Patients were divided into four quartile subgroups based on rescue medication use (Q1: patients in the $<25$ percentile of rescue medication use, Q2: patients in the 25$<50$ percentile, Q3: patients in the $50-<75$ percentile, Q4: patients in the $\geq 75$ percentile of rescue medication use); details on rescue medication use in the quartile subgroups are shown in Table 1. Of note, the rescue quartiles were determined using all subjects who had baseline rescue medication use data in the two studies, including those patients who received GLY $50 \mu \mathrm{g}$ BID.

Patient baseline demographics and disease characteristics are shown in Table 2. Patients in the Q1 subgroup were oldest, with median age being similar across all other rescue medication use subgroups. Use of background LABA and inhaled corticosteroids (ICS) was greater with increasing baseline rescue medication use, independent of treatment arm; similarly, baseline lung function and PRO scores were worse with increasing rescue medication use, except for baseline $\mathrm{FEV}_{1}$ in the Q3 subgroup, which was similar to the values observed in the Q1 subgroup. The history of COPD exacerbations in the 12 months prior to the studies was lowest in patients receiving GLY $25 \mu \mathrm{g}$ BID in the Q1 subgroup but similar across all other treatment groups and rescue medication use subgroups. In addition, the proportion of current smokers was lowest in the Q1 subgroup, and similar across other subgroups; however, the number of pack-years was highest among Q1 subgroup and similar across other subgroups.

\section{Efficacy}

Change from Baseline in SGRQ and EXACT-RS Total Scores

Improvements from baseline in SGRQ total score with GLY

$25 \mu \mathrm{g}$ BID at 12 weeks were significantly greater than placebo in all baseline rescue medication subgroups except the Q3 subgroup ( $p=0.340$; Figure $2 \mathrm{~A}$ ). In contrast, improvements in the SGRQ three domain scores were variable across the rescue medication quartiles (Figure 2B-D). Patients treated with GLY $25 \mu \mathrm{g}$ BID in the Q4 subgroup reported a clinically significant $(\geq 4$-unit reduction from baseline) improvement in SGRQ total score (Figure 2A). The odds of being an SGRQ responder ( $\geq 4$-unit reduction) in the GLY treatment group were only significant in the Q4 subgroup $(p<0.05)$ but were numerically higher with GLY compared with placebo in all other baseline rescue medication subgroups (Figure 2E).
The placebo-adjusted changes from baseline in EXACT-RS total score with GLY treatment at 12 weeks were significant only in the Q1 baseline rescue medication subgroup (Figure 3A). However, the odds of being an EXACT-RS responder ( $\geq 2$-unit reduction) in the GLY treatment group were only significant in the Q4 subgroup $(p<0.05)$ but were numerically higher than placebo in all other baseline rescue medication subgroups (Figure 3B).

\section{Changes from Baseline in Lung Function}

At 12 weeks, treatment with GLY led to numerically greater improvements from baseline in trough $\mathrm{FEV}_{1}$ compared with placebo in all rescue medication use subgroups (Figure 4), with clinically important differences observed among patients in the Q1 and Q3 subgroups. Placeboadjusted improvements from baseline in trough $\mathrm{FEV}_{1}$ were significant in all rescue medication subgroups, except for Q4 ( $p=0.087$; Figure 4).

\section{Changes from Baseline in Rescue Medication Use}

Over 12 weeks, changes from baseline in rescue medication use showed a similar pattern with both placebo and GLY treatment (Figure 5). The number of daily rescue medication puffs showed similar, minimal changes from baseline over 12 weeks in the Q1 and Q2 subgroups (Q1: placebo, 0.20, GLY, 0.20; Q2: placebo: -0.15, GLY, -0.09; Figure 5) while greater changes from baseline were observed with placebo and GLY over 12 weeks in patients in the Q3 and Q4 subgroups (Q3: placebo, -0.69, GLY, -1.02; Q4: placebo, -1.78 , GLY, -2.17 ; Figure 5). There were no significant differences in the number of daily puffs of rescue medication over 12 weeks between placebo and GLY (Figure 5).

\section{Safety}

Overall, GLY was generally well tolerated, regardless of the baseline rescue medication use, with similar incidence of AEs across the different subgroups (Table 3). The overall incidence rate of AEs was lower with GLY compared to placebo in all rescue medication subgroups except the Q3 patients. The most common AEs in all rescue medication use subgroups were worsening of COPD, cough, and dyspnea (Table 3). There was lower incidence of any SAEs among patients receiving GLY compared with placebo in all rescue medication use subgroups.

\section{Discussion}

Rescue medication use is common among patients with COPD seeking to alleviate respiratory symptoms or 


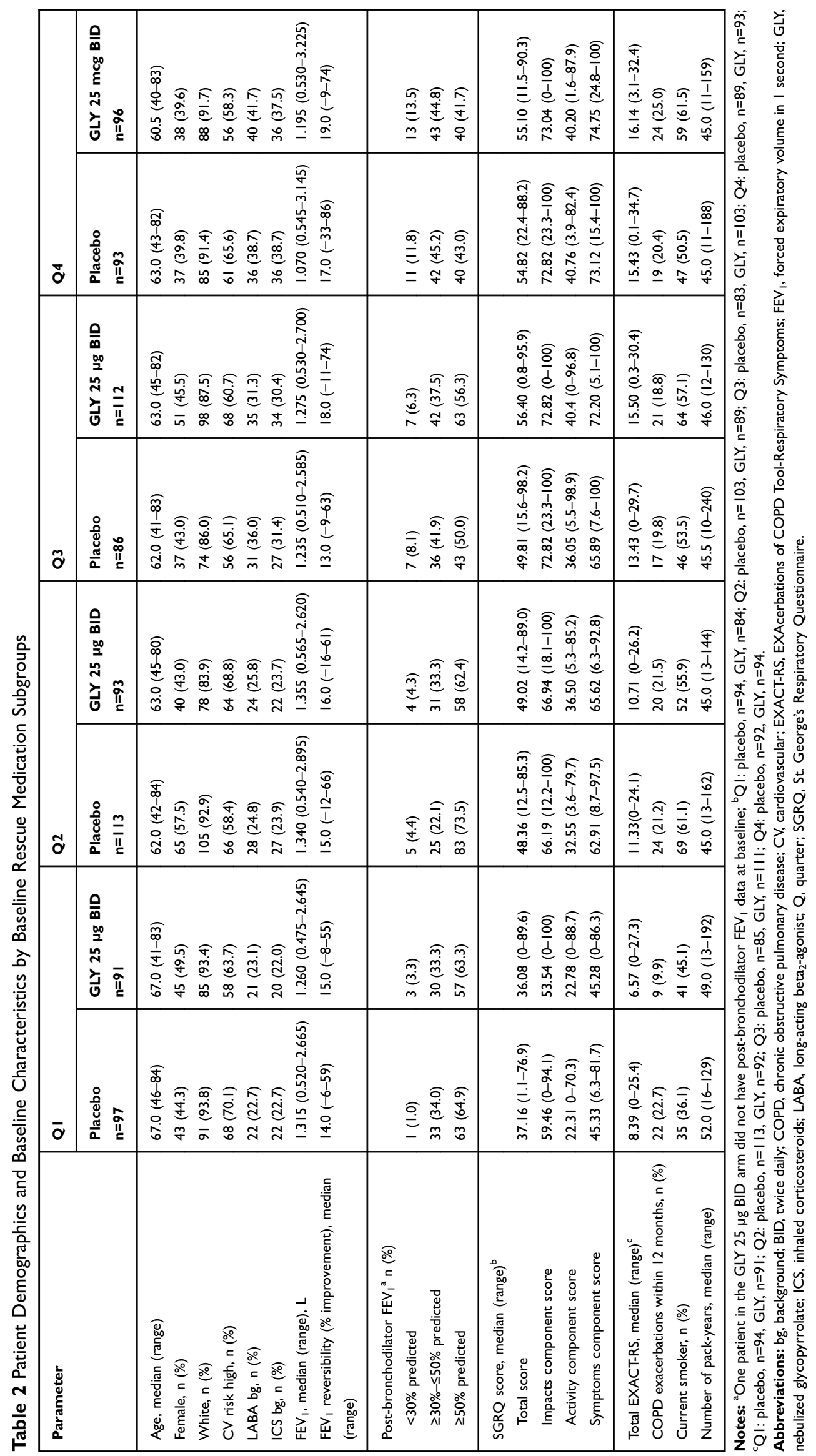



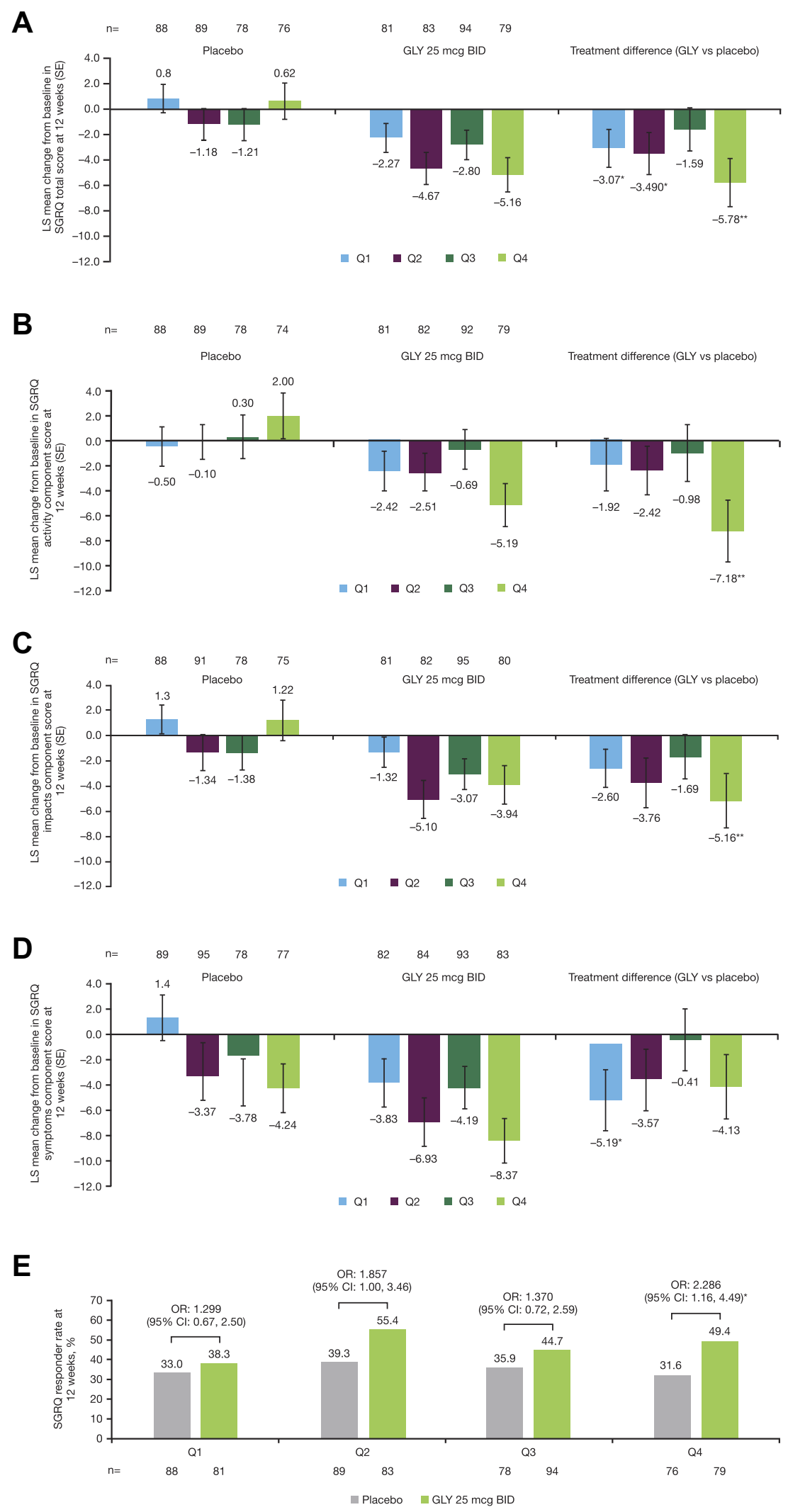

Figure 2 Pooled analysis of change from baseline in SGRQ (A) total score, (B) activity, (C) impacts, (D) symptoms component scores, and (E) responders at I2 weeks, by baseline rescue medication use (ITT population).

Notes: $* P<0.05 ; * * P<0.01$ versus placebo; $n$-values represent the number of patients with on-treatment data at week 12 .

Abbreviations: BID, twice daily; CI, confidence interval; GLY, nebulized glycopyrrolate; ITT, intent-to-treat; LS, least squares; OR, odds ratio; Q, quarter; SE, standard error; SGRQ, St. George's Respiratory Questionnaire. 


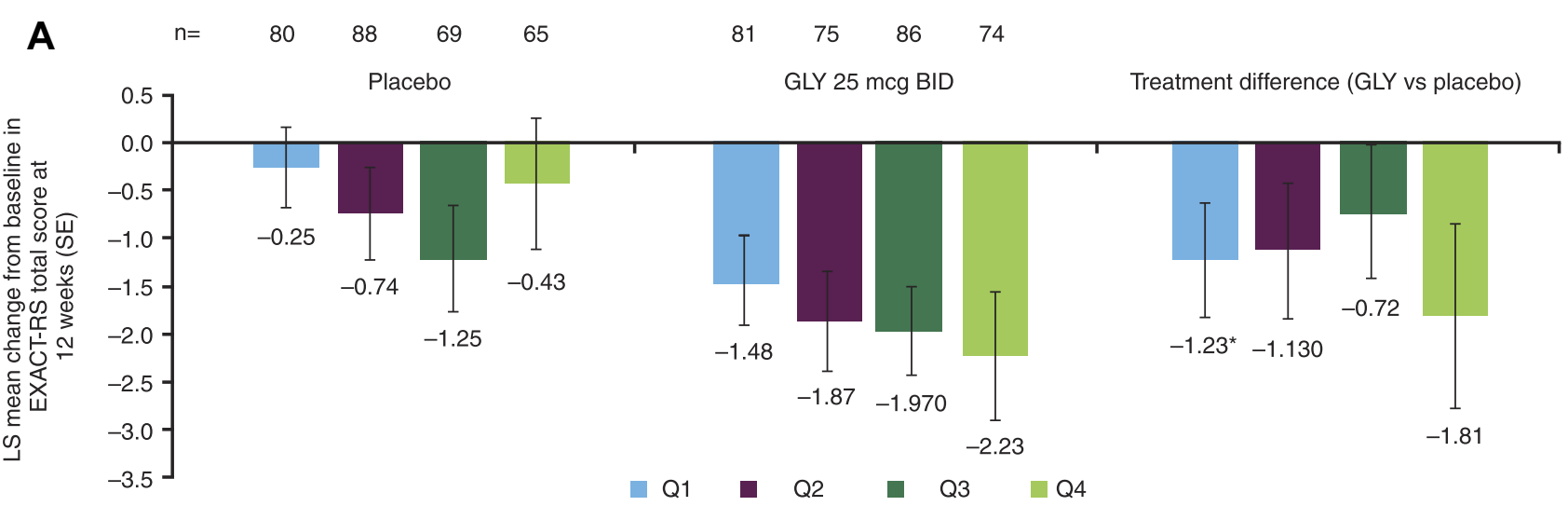

B

OR: 1.257

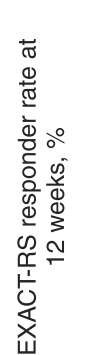

OR: 1.661

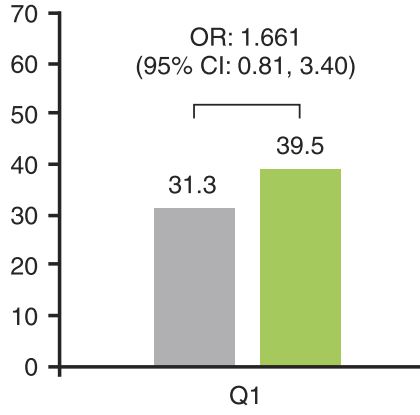

$\mathrm{n}=$

80

81
(95\% Cl: $0.66,2.38)$

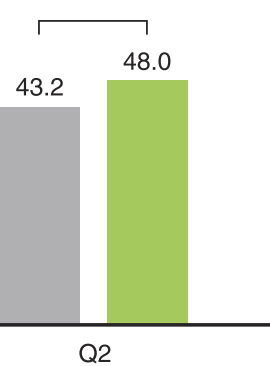

88
OR: 1.457

(95\% Cl: $0.78,2.73)$

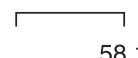

OR: 2.234

(95\% Cl: $1.13,4.41)^{\star}$

58.1

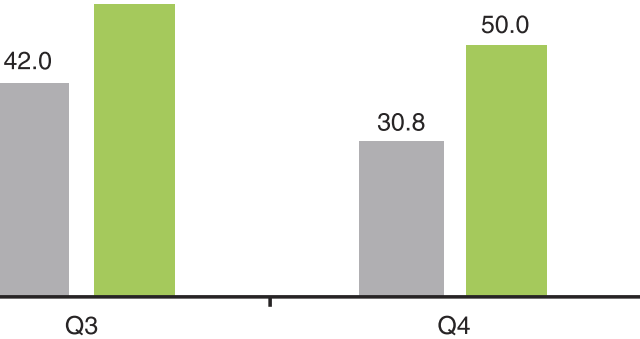

$69 \quad 86$

65

74

Figure 3 Pooled analysis of change from baseline in EXACT-RS (A) total score and (B) responders at 12 weeks, by baseline rescue medication use (ITT population). Notes: $* P<0.05$ versus placebo; $n$-values represent the number of patients with on-treatment data at week 12.

Abbreviations: BID, twice daily; Cl, confidence interval; EXACT-RS, EXAcerbations of COPD Tool-Respiratory Symptoms; GLY, nebulized glycopyrrolate; ITT, intent-totreat; LS, least squares; OR, odds ratio; $\mathrm{Q}$, quarter; SE, standard error.

\begin{tabular}{cccrr}
$n=$ & 86 & 64 & 60 \\
& \multicolumn{4}{c}{ Placebo }
\end{tabular}

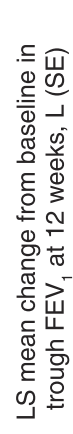

$-0.10$

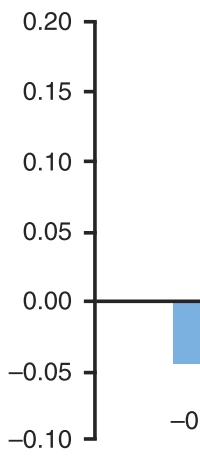
82
$72 \quad 89$
76
GLY 25 mcg BID

Treatment difference (GLY vs placebo)

$0.152^{* * *}$

Figure 4 Pooled analysis of change from baseline in trough $\mathrm{FEV}_{\mathrm{I}}$ at 12 weeks by baseline rescue medication use subgroup (ITT population).

Notes: ${ }^{*} p<0.05,{ }^{*} p<0.01,{ }^{* * *} p<0.001$ versus placebo; $n$-values represent the number of patients with on-treatment data at week 12 .

Abbreviations: BID, twice daily; FEV 1 , forced expiratory volume in I second; GLY, nebulized glycopyrrolate; ITT, intent-to-treat; LS, least squares; Q, quarter; SE, standard error. 


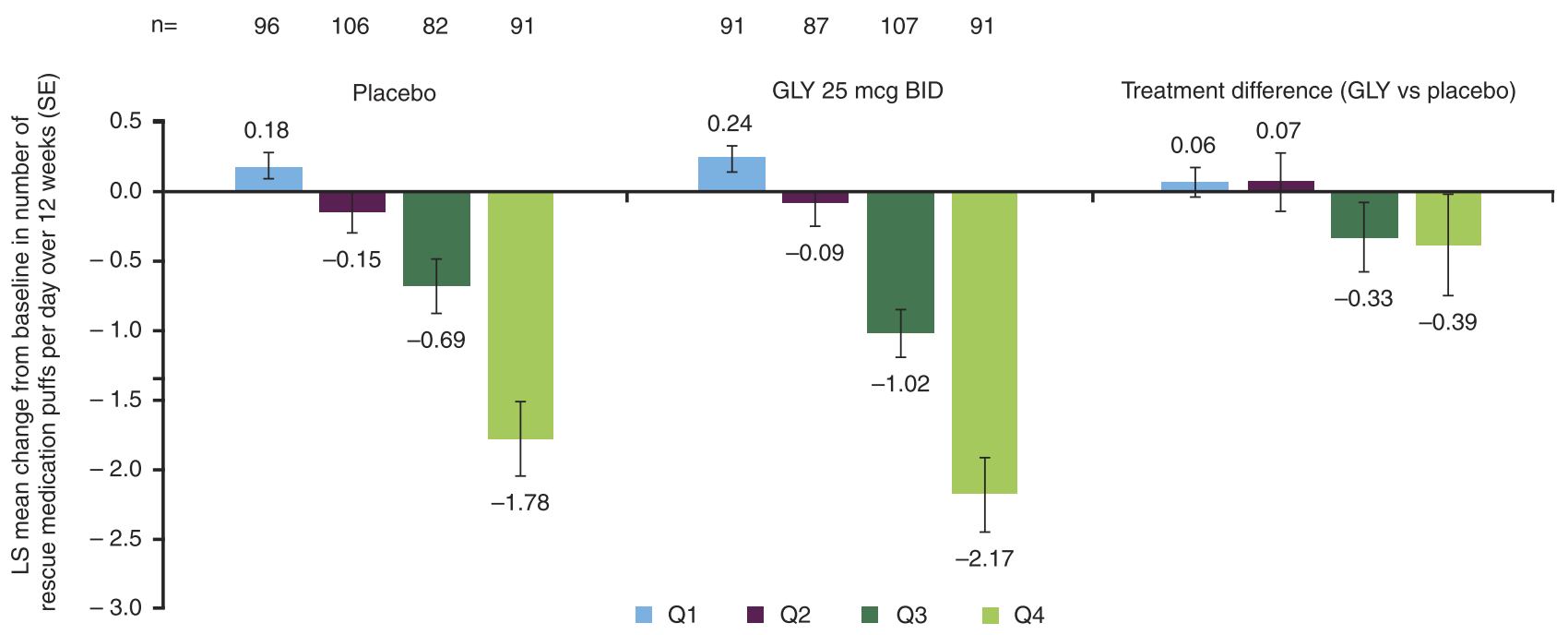

Figure 5 Pooled analysis of change from baseline in the number of puffs of rescue medication per day over 12 weeks by baseline rescue medication use subgroup (ITT population). Note: $n$-values represent the number of patients with on-treatment data at week 12.

Abbreviations: BID, twice daily; GLY, nebulized glycopyrrolate; ITT, intent-to-treat; LS, least squares; Q, quarter; SE, standard error.

control acute exacerbations. ${ }^{1}$ The frequency and extent of rescue medication use are known to correlate with more severe disease and increased exacerbations. While there are limited analyses of the impact of baseline rescue medication use on efficacy and safety outcomes of long-acting bronchodilators in patients with COPD, one analysis of QVA149 based on rescue medication use showed no differences in improvements of lung function or PROs compared with placebo or comparator drug. ${ }^{4}$ These results are consistent with those observed in this analysis of pooled data from the GOLDEN 3 and GOLDEN 4 studies which showed that baseline rescue medication use did not affect improvements in lung function or alter the safety profile of GLY compared with placebo. Improvements in SGRQ, EXACT-RS and lung function were aligned and tended to be greater with higher rescue medication use at baseline. An important distinction between the prior analysis ${ }^{4}$ and the current study is that our analysis stratified patients into subgroups of rescue medication use, thereby allowing further in-depth investigation of the effects of different levels of baseline rescue medication use on treatment outcomes.

Table 3 Summary of AEs and SAEs, Including Individual AEs with Incidence $\geq 3 \%$ in Any Treatment Group, by Baseline Rescue Medication Use Subgroup (Safety Population)

\begin{tabular}{|c|c|c|c|c|c|c|c|c|}
\hline \multirow[t]{2}{*}{ Preferred Term, n (\%) } & \multicolumn{2}{|l|}{ QI } & \multicolumn{2}{|l|}{ Q2 } & \multicolumn{2}{|l|}{ Q3 } & \multicolumn{2}{|l|}{ Q4 } \\
\hline & $\begin{array}{l}\text { Placebo } \\
\text { n=97 }\end{array}$ & $\begin{array}{l}\text { GLY } 25 \mu g \\
\text { BID } \\
n=9 \text { I }\end{array}$ & $\begin{array}{l}\text { Placebo } \\
n=113\end{array}$ & $\begin{array}{l}\text { GLY } 25 \mu \mathrm{g} \\
\text { BID } \\
n=93\end{array}$ & $\begin{array}{l}\text { Placebo } \\
n=86\end{array}$ & $\begin{array}{l}\text { GLY } 25 \mu g \text { BID } \\
n=1 / 2\end{array}$ & $\begin{array}{l}\text { Placebo } \\
n=93\end{array}$ & $\begin{array}{l}\text { GLY } 25 \mu g \\
\text { BID } \\
n=96\end{array}$ \\
\hline Any AE & $51(52.6)$ & $33(36.3)$ & $60(53.1)$ & $42(45.2)$ & $42(48.8)$ & $56(50.0)$ & $50(53.8)$ & $38(39.6)$ \\
\hline Cough & $14(14.4)$ & $\mathrm{I}(\mathrm{I} . \mathrm{I})$ & $11(9.7)$ & $10(10.8)$ & $4(4.7)$ & $9(8.0)$ & $2(2.2)$ & $6(6.3)$ \\
\hline Worsening of COPD & $6(6.2)$ & $4(4.4)$ & $9(8.0)$ & $5(5.4)$ & $4(4.7)$ & $9(8.0)$ & $15(16.1)$ & $5(5.2)$ \\
\hline Dyspnea & $2(2.1)$ & $2(2.2)$ & $6(5.3)$ & $5(5.4)$ & $\mathrm{I}(1.2)$ & $8(7.1)$ & $3(3.2)$ & $5(5.2)$ \\
\hline Upper respiratory tract infection & 0 & $3(3.3)$ & I (0.9) & $\mathrm{I}(\mathrm{I.I})$ & $3(3.5)$ & $2(1.8)$ & 0 & 0 \\
\hline Nasopharyngitis & 0 & $4(4.4)$ & 0 & $\mathrm{I}(\mathrm{l} .1)$ & $2(2.3)$ & $2(1.8)$ & $2(2.2)$ & 0 \\
\hline Urinary tract infection & 0 & 0 & $3(2.7)$ & $3(3.2)$ & $\mathrm{I}(1.2)$ & $2(1.8)$ & $2(2.2)$ & $4(4.2)$ \\
\hline Headache & $2(2.1)$ & $3(3.3)$ & I (0.9) & $\mathrm{I}(\mathrm{I.1})$ & $4(4.7)$ & 0 & $2(2.2)$ & I (I.0) \\
\hline Arthralgia & $3(3.1)$ & $\mathrm{I}(\mathrm{I} .1)$ & $2(1.8)$ & $\mathrm{I}(\mathrm{l} .1)$ & 0 & 0 & 0 & I (I.0) \\
\hline Gastroenteritis & $\mathrm{I}(1.0)$ & 0 & 0 & 0 & $2(2.3)$ & I (0.9) & $3(3.2)$ & I (I.0) \\
\hline Diarrhea & I (I.0) & 0 & I (0.9) & 0 & 0 & I (0.9) & $3(3.2)$ & 0 \\
\hline Edema peripheral & 0 & $\mathrm{I}(\mathrm{I} .1)$ & 0 & $\mathrm{I}(\mathrm{I} .1)$ & 0 & I (0.9) & $3(3.2)$ & $I(1.0)$ \\
\hline Any SAE & $5(5.2)$ & $3(3.3)$ & $5(4.4)$ & $2(2.2)$ & $4(4.7)$ & $4(3.6)$ & $7(7.5)$ & $3(3.1)$ \\
\hline
\end{tabular}

Abbreviations: AE, adverse event; BID, twice daily; COPD, chronic obstructive pulmonary disease; GLY, nebulized glycopyrrolate; Q, quarter; SAE, serious adverse event. 
Patient baseline characteristics in this analysis showed some differences associated with disease and symptom severity between the subgroups. Background use of LABA and ICS was greater in subgroups with higher baseline rescue medication use, whereas lung function and PRO scores were generally worse in subgroups with higher baseline rescue medication use. These outcomes are to be expected, as rescue medication use correlates with worse symptoms and disease severity, and serve to further underscore the significance of the current analysis to determine potential differences in treatment response based on baseline rescue medication use.

In this analysis, changes in both SGRQ and EXACTRS total scores suggested greater improvements from baseline with greater baseline rescue medication use. However, it is important to note that the significance of these improvements relative to placebo were only detected among patients receiving GLY in some, but not all, of the rescue medication subgroups. This may, in part, be attributable to differences in placebo responses in the subgroups, which may in turn be a result of the clear differences in patient baseline characteristics. Importantly, changes from baseline were numerically greater with GLY compared with placebo in all rescue medication subgroups, and the proportion of SGRQ and EXACT-RS responders was greater with GLY than placebo in all rescue medication subgroups. These outcomes support the improvements in PROs observed with GLY treatment in all rescue medication use subgroups, but suggest that the magnitude and significance of PRO improvements may vary with the level of baseline rescue medication use.

Lung function improvement from baseline with GLY was similar across all baseline rescue medication subgroups. However, significant differences relative to placebo were observed in all subgroups except for the Q4 subgroup; this lack of significance in the subgroup of patients having the highest baseline rescue medication use may be a result of the greater disease severity or symptomology in these patients, which may have resulted in decreased functional improvements.

The changes in rescue medication use with GLY treatment were similar to those with placebo, regardless of baseline rescue medication use subgroup. This is consistent with the results seen in the primary analysis of the GOLDEN 3/4 studies, wherein no significant differences in rescue medication use were observed between placebo and GLY. ${ }^{7}$

GLY was generally well tolerated across the rescue medication use subgroups and no new safety signals were identified. Overall, the subgroup safety profiles were consistent with the safety profiles from the two 12week pivotal studies. ${ }^{7}$

Limitations to the current analysis include the post-hoc patient stratification based on baseline rescue medication use and the lack of control for multiplicity. Therefore, differences in patient baseline and disease characteristics between the subgroups which could not be controlled for may have impacted the observed outcomes. In addition, while the distribution of patients into quartiles based on rescue medication use utilized the entire patient populations (ie, placebo, 25 and $50 \mu \mathrm{g}$ treatment groups) of the two 12-week pivotal studies for modeling, the data for the GLY $50 \mu \mathrm{g}$ BID dose were excluded and the results presented here are for the FDA-approved dose of GLY, ie, $25 \mu \mathrm{g} \mathrm{BID} .^{6}$

\section{Conclusions}

The value of baseline rescue medication use as a predictor of response to bronchodilators among patients with COPD has not been explored. In this analysis of pooled results from GOLDEN 3/4 studies stratified by baseline rescue medication use, patients treated with GLY demonstrated improvements in lung function, SGRQ total score, and EXACT-RS total score compared with placebo, regardless of background rescue medication use. In addition, treatment with GLY was generally well tolerated across all of the rescue medication subgroups. These data support the efficacy and safety of GLY $25 \mu \mathrm{g}$ BID in patients with moderate-to-very-severe $\mathrm{COPD},{ }^{7,11}$ independent of baseline rescue medication use. The findings of this analysis suggest that the frequency of rescue medication use may provide relevant information to clinicians related to COPD symptoms and control, especially when spirometry and symptom scale assessments are unavailable.

\section{Data Sharing Statement}

Sunovion Pharmaceuticals Inc. is part of a clinical trial data sharing consortium that facilitates access for qualified researchers to selected anonymized clinical trial data. For up-to-date information on data availability, please visit https://www.clinicalstudydatarequest.com/Study-Sponsors. aspx and click on Sunovion.

\section{Acknowledgments}

The authors would like to thank Shane Hornibrook from Sarepta Therapeutics Inc., Diane Hall from Sunovion Pharmaceuticals, Inc., and Rajeshwari Sammishetty from 
Sage Therapeutic, Inc., for support with statistical analyses performed. This post-hoc analysis was funded by Sunovion Pharmaceuticals, Inc. Medical writing support was provided by Hashem Dbouk, PhD, of FireKite, an Ashfield company, part of UDG Healthcare plc, and funded by Sunovion Pharmaceuticals, Inc. The abstract of this paper was presented at the American Thoracic Society 2019 International Conference as a poster with interim findings. The poster's abstract was published in the "Abstracts Issue" of American Journal of Respiratory and Critical Care Medicine: https://doi.org/10.1164/ajrccm-conference.2019. 199.1_MeetingAbstracts.A3873.

\section{Disclosure}

JFD has been an advisor and received personal fees from Sunovion and Mylan, and has served on advisory boards for Theravance. AO-G, TG, and SS are employees of Sunovion Pharmaceuticals, Inc. The authors report no other conflicts of interest in this work.

\section{References}

1. Global Initiative for Chronic Obstructive Lung Disease (GOLD). Global strategy for the diagnosis, management and prevention of COPD, 2020. Available from: http://goldcopd.org/. Accessed February 12, 2020.

2. Jenkins CR, Postma DS, Anzueto AR, et al. Reliever salbutamol use as a measure of exacerbation risk in chronic obstructive pulmonary disease. BMC Pulm Med. 2015;15:97. doi:10.1186/s12890-015-0077-0
3. Punekar YS, Sharma S, Pahwa A, Takyar J, Naya I, Jones PW. Rescue medication use as a patient-reported outcome in COPD: a systematic review and regression analysis. Respir Res. 2017;18 (1):86. doi:10.1186/s12931-017-0566-1

4. Chapman KR, Bateman ED, Chen H, Hu H, Fogel R, Banerji D. QVA149 improves lung function, dyspnea, and health status independent of previously prescribed medications and COPD severity: a subgroup analysis from the SHINE and ILLUMINATE studies. Chronic Obstr Pulm Dis. 2015;2(1):48-60. doi:10.15326/ jcopdf.2.1.2014.0140

5. Pham S, Ferguson GT, Kerwin E, Goodin T, Wheeler A, Bauer A. In vitro characterization of the eFlow closed system nebulizer with glycopyrrolate inhalation solution. J Aerosol Med Pulm Drug Deliv. 2018;31(3):162-169. doi:10.1089/jamp.2017.1384

6. Lonhala Magnair (glycopyrrolate) inhalation solution [highlights of prescribing information]. Sunovion Pharmaceuticals, Inc; 2019

7. Kerwin E, Donohue JF, Goodin T, Tosiello R, Wheeler A, Ferguson GT. Efficacy and safety of glycopyrrolate/eFlow ${ }^{\circledR}$ CS (nebulized glycopyrrolate) in moderate-to-very-severe COPD: results from the glycopyrrolate for obstructive lung disease via electronic nebulizer (GOLDEN) 3 and 4 randomized controlled trials. Respir Med. 2017;132:238-250. doi:10.1016/j.rmed.2017.07.011

8. Global Initiative for Chronic Obstructive Lung Disease (GOLD). Global strategy for the diagnosis, management and prevention of COPD, 2014. Available from: http://goldcopd.org/. Accessed May $20,2019$.

9. Jones PW. St. George's respiratory questionnaire: MCID. COPD. 2005;2(1):75-79. doi:10.1081/COPD-200050513

10. Leidy NK, Murray LT, Monz BU, et al. Measuring respiratory symptoms of COPD: performance of the EXACT- Respiratory Symptoms Tool (E-RS) in three clinical trials. Respir Res. 2014;15:124. doi:10.1186/s12931-014-0124-z

11. Kerwin E, Ferguson GT. An overview of glycopyrrolate/eFlow ${ }^{\circledR}$ CS in COPD. Expert Rev Respir Med. 2018;12(6):447-459. doi:10.1080/ 17476348.2018.1476853

\section{Publish your work in this journal}

The International Journal of COPD is an international, peer-reviewed journal of therapeutics and pharmacology focusing on concise rapid reporting of clinical studies and reviews in COPD. Special focus is given to the pathophysiological processes underlying the disease, intervention programs, patient focused education, and self management protocols. This journal is indexed on PubMed Central, MedLine and CAS. The manuscript management system is completely online and includes a very quick and fair peer-review system, which is all easy to use. Visit http://www.dovepress.com/testimonials.php to read real quotes from published authors. 